\title{
An Investigation on the Beta Function I: New Versions of the Euler Beta Function \\ Edigles Guedes ${ }^{1}$ and Prof. Dr. K. Raja Rama Gandhi ${ }^{2}$
}

Number Theorist, Brazil ${ }^{1}$

Resource perosn in Mathematics for Oxford University Press and Professor at BITS-Vizag ${ }^{2}$ Abstract. I developed new versions of the Euler beta function.

\section{Introduction}

In this paper, I discovered the formulas, among others:

$$
\begin{gathered}
\frac{1}{\pi}=-\frac{4 \Gamma\left(\frac{1}{3}\right)}{5 \Gamma\left(\frac{4}{3}\right) \Gamma\left(\frac{5}{6}\right) \Gamma\left(-\frac{5}{6}\right)} \\
\frac{1}{\pi}=\frac{1}{\left[\frac{1}{8}(1+\sqrt{5})-\frac{1}{4} \sqrt{\left.\frac{3}{2}(5-\sqrt{5})\right] \Gamma\left(-\frac{1}{30}\right) \Gamma\left(\frac{31}{30}\right)}\right.}, \\
\frac{1}{\pi}=-\frac{2}{\Gamma\left(-\frac{5}{6}\right) \Gamma\left(\frac{11}{6}\right)}
\end{gathered}
$$

and

$$
\frac{1}{\pi}=\frac{2 \sqrt{2}}{(1-\sqrt{3}) \Gamma\left(-\frac{1}{12}\right) \Gamma\left(\frac{13}{12}\right)}
$$

\section{THEOREM}

Theorem 1. For $\mathfrak{R}(x)>0$ and $\mathfrak{R}(y)>0$, then

$$
\frac{\Gamma(x) \Gamma(y)}{\Gamma(x+y) \Gamma\left(y+\frac{1}{2}\right)}=\sum_{k=0}^{\infty} \frac{(2 k) ! \Gamma(x+k)}{4^{k} k !^{2} \Gamma\left(x+y+k+\frac{1}{2}\right)} .
$$

Proof. In [1, equation 7], I have

$$
\frac{1}{\sqrt{1-z^{2}}}=\sum_{k=0}^{\infty} \frac{(2 k) !}{4^{k} k !^{2}} z^{2 k}
$$

consequently,

$$
\frac{1}{\sqrt{1-t}}=\sum_{k=0}^{\infty} \frac{(2 k) !}{4^{k} k !^{2}} t^{k}
$$

On the other hand, [2, page 898], I encounter the definition

$$
B(x, y) \stackrel{\text { def }}{=} \int_{0}^{1} t^{x-1}(1-t)^{y-1} \mathrm{~d} t
$$


I did the following algebraic manipulation in the above definition

$$
B(x, y)=\int_{0}^{1} \frac{t^{x-1}(1-t)^{y-\frac{1}{2}}}{\sqrt{1-t}} \mathrm{~d} t .
$$

I substitute (2) into (4), and obtain

$$
\begin{gathered}
B(x, y)=\int_{0}^{1} t^{x-1}(1-t)^{y-\frac{1}{2}} \sum_{k=0}^{\infty} \frac{(2 k) !}{4^{k} k !^{2}} t^{k} \mathrm{~d} t \\
=\sum_{k=0}^{\infty} \frac{(2 k) !}{4^{k} k !^{2}} \int_{0}^{1} t^{x+k-1}(1-t)^{y-\frac{1}{2}} \mathrm{~d} t \\
=\sum_{k=0}^{\infty} \frac{(2 k) !}{4^{k} k !^{2}} \cdot \frac{\Gamma\left(y+\frac{1}{2}\right) \Gamma(x+k)}{\Gamma\left(x+y+k+\frac{1}{2}\right)} \\
=\Gamma\left(y+\frac{1}{2}\right) \sum_{k=0}^{\infty} \frac{(2 k) ! \Gamma(x+k)}{4^{k} k !^{2} \Gamma\left(x+y+k+\frac{1}{2}\right)} .
\end{gathered}
$$

In [2, page 899], I meet

$$
B(x, y)=\frac{\Gamma(x) \Gamma(y)}{\Gamma(x+y)}
$$

From (5) and (6), I take

$$
\frac{\Gamma(x) \Gamma(y)}{\Gamma(x+y) \Gamma\left(y+\frac{1}{2}\right)}=\sum_{k=0}^{\infty} \frac{(2 k) ! \Gamma(x+k)}{4^{k} k !^{2} \Gamma\left(x+y+k+\frac{1}{2}\right)} .
$$

Corollary 1. For $\mathfrak{R}(y)>0$, then

$$
\frac{\Gamma(y)}{\Gamma(y+1) \Gamma\left(y+\frac{1}{2}\right)}=\sum_{k=0}^{\infty} \frac{(2 k) !}{4^{k} k ! \Gamma\left(y+k+\frac{3}{2}\right)}
$$

Proof. Let $x=1$ and this completes the proof. $\square$

Corollary 2. For $\mathfrak{R}(x)>0$ and $\mathfrak{R}(y)>0$, then

$$
\begin{gathered}
\frac{1}{\pi}=\frac{1}{\cos (\pi(x+y)) \Gamma\left(-x-y+\frac{1}{2}\right) \Gamma\left(x+y+\frac{1}{2}\right)}, \\
\frac{1}{\pi}=\frac{-1}{\cos (\pi y) \Gamma\left(-y-\frac{1}{2}\right) \Gamma\left(y+\frac{3}{2}\right)}
\end{gathered}
$$

and

$$
\Gamma(x) \Gamma(-x)=-\frac{\pi}{x \sin (\pi x)}
$$

Proof. In [2, page 887], I have 


$$
\Gamma\left(\frac{1}{2}+z\right) \Gamma\left(\frac{1}{2}-z\right)=\frac{\pi}{\cos (\pi z)}
$$

hence,

$$
\frac{1}{\Gamma\left(z+\frac{1}{2}\right)}=\Gamma\left(\frac{1}{2}-z\right) \frac{\cos (\pi z)}{\pi}
$$

I substitute (8) into Theorem 1 and Corollary 1, separately.

$$
\frac{\pi \Gamma(x) \Gamma(y)}{\Gamma(x+y) \Gamma\left(y+\frac{1}{2}\right)}=\sum_{k=0}^{\infty} \frac{(2 k) ! \cos [\pi(x+y+k)] \Gamma(x+k) \Gamma\left(\frac{1}{2}-x-y-k\right)}{4^{k} k !^{2}},
$$

so

$$
\begin{gathered}
\frac{\pi \Gamma(x) \Gamma(y)}{\Gamma(x+y) \Gamma\left(y+\frac{1}{2}\right)}=\frac{\Gamma(x) \Gamma(y) \cos (\pi(x+y)) \Gamma\left(-x-y+\frac{1}{2}\right) \Gamma\left(x+y+\frac{1}{2}\right)}{\Gamma(x+y) \Gamma\left(y+\frac{1}{2}\right)} \Rightarrow \\
\frac{1}{\pi}=\frac{1}{\cos (\pi(x+y)) \Gamma\left(-x-y+\frac{1}{2}\right) \Gamma\left(x+y+\frac{1}{2}\right)}
\end{gathered}
$$

and

$$
\frac{\pi \Gamma(y)}{\Gamma(y+1) \Gamma\left(y+\frac{1}{2}\right)}=\sum_{k=0}^{\infty} \frac{(2 k) ! \Gamma\left(-\frac{1}{2}-y-k\right) \cos [\pi(y+k+1)]}{4^{k} k !}
$$

so

$\frac{\pi \Gamma(y)}{\Gamma(y+1) \Gamma\left(y+\frac{1}{2}\right)}=-\frac{\cos (\pi y) \Gamma\left(-y-\frac{1}{2}\right) \Gamma(y) \Gamma\left(y+\frac{3}{2}\right)}{\Gamma(y+1) \Gamma\left(y+\frac{1}{2}\right)} \Rightarrow \frac{1}{\pi}=\frac{-1}{\cos (\pi y) \Gamma\left(-y-\frac{1}{2}\right) \Gamma\left(y+\frac{3}{2}\right)}$

I take $y=\frac{1}{2}$ in $(8)$, and have

$$
\Gamma(x+1) \Gamma(-x)=\frac{\pi}{\cos \left(\pi\left(x+\frac{1}{2}\right)\right)}=-\frac{\pi}{\sin (\pi x)} .
$$

On the other hand, $\Gamma(x+1)=x \Gamma(x)$, that subsitituting in the previous equation gives the desired result. $\square$

Corollary 3. I have

$$
\begin{gathered}
\Gamma\left(\frac{1}{30}\right)=30 \Gamma\left(\frac{31}{30}\right), \\
\Gamma\left(\frac{1}{3}\right)=3 \Gamma\left(\frac{4}{3}\right), \\
\Gamma\left(\frac{1}{12}\right)=12 \Gamma\left(\frac{13}{12}\right),
\end{gathered}
$$




$$
\begin{gathered}
\frac{1}{\pi}=-\frac{4 \Gamma\left(\frac{1}{3}\right)}{5 \Gamma\left(\frac{4}{3}\right) \Gamma\left(\frac{5}{6}\right) \Gamma\left(-\frac{5}{6}\right)}, \\
\frac{1}{\pi}=\frac{1}{\left[\frac{1}{8}(1+\sqrt{5})-\frac{1}{4} \sqrt{\frac{3}{2}(5-\sqrt{5})}\right] \Gamma\left(-\frac{1}{30}\right) \Gamma\left(\frac{31}{30}\right)}, \\
\frac{1}{\pi}=-\frac{2}{\Gamma\left(-\frac{5}{6}\right) \Gamma\left(\frac{11}{6}\right)}
\end{gathered}
$$

and

$$
\frac{1}{\pi}=\frac{2 \sqrt{2}}{(1-\sqrt{3}) \Gamma\left(-\frac{1}{12}\right) \Gamma\left(\frac{13}{12}\right)} .
$$

Proof. Let $x=\frac{1}{3}$ and $y=\frac{1}{5}$ in Theorem 1, then

$$
\frac{\Gamma\left(\frac{1}{3}\right) \Gamma\left(\frac{1}{5}\right)}{\Gamma\left(\frac{8}{15}\right) \Gamma\left(\frac{7}{10}\right)}=\sum_{k=0}^{\infty} \frac{(2 k) ! \Gamma\left(\frac{1}{3}+k\right)}{4^{k} k !^{2} \Gamma\left(\frac{31}{30}+k\right)}=\frac{30 \Gamma\left(\frac{1}{5}\right) \Gamma\left(\frac{1}{3}\right) \Gamma\left(\frac{31}{30}\right)}{\Gamma\left(\frac{1}{30}\right) \Gamma\left(\frac{8}{15}\right) \Gamma\left(\frac{7}{10}\right)} \Rightarrow \Gamma\left(\frac{1}{30}\right)=30 \Gamma\left(\frac{31}{30}\right) .
$$

Let $x=\frac{1}{3}$ and $y=\frac{1}{2}$ in Theorem 1, then

$$
\frac{\Gamma\left(\frac{1}{3}\right) \sqrt{\pi}}{\Gamma\left(\frac{5}{6}\right)}=\sum_{k=0}^{\infty} \frac{(2 k) ! \Gamma\left(\frac{1}{3}+k\right)}{4^{k} k !^{2} \Gamma\left(\frac{4}{3}+k\right)}=\frac{3 \sqrt{\pi} \Gamma\left(\frac{4}{3}\right)}{\Gamma\left(\frac{5}{6}\right)} \Rightarrow \Gamma\left(\frac{1}{3}\right)=3 \Gamma\left(\frac{4}{3}\right) .
$$

Let $x=\frac{1}{3}$ and $y=\frac{1}{4}$ in Theorem 1, then

$$
\frac{\Gamma\left(\frac{1}{3}\right) \Gamma\left(\frac{1}{4}\right)}{\Gamma\left(\frac{7}{12}\right) \Gamma\left(\frac{3}{4}\right)}=\sum_{k=0}^{\infty} \frac{(2 k) ! \Gamma\left(\frac{1}{3}+k\right)}{4^{k} k !^{2} \Gamma\left(\frac{13}{12}+k\right)}=\frac{12 \Gamma\left(\frac{1}{4}\right) \Gamma\left(\frac{1}{3}\right) \Gamma\left(\frac{13}{12}\right)}{\Gamma\left(\frac{1}{12}\right) \Gamma\left(\frac{7}{12}\right) \Gamma\left(\frac{3}{4}\right)} \Rightarrow \Gamma\left(\frac{1}{12}\right)=12 \Gamma\left(\frac{13}{12}\right) \text {. }
$$

Let $y=\frac{1}{3}$ in Corollary 2, then

$$
\begin{gathered}
\frac{\pi \Gamma\left(\frac{1}{3}\right)}{\Gamma\left(\frac{4}{3}\right) \Gamma\left(\frac{5}{6}\right)}=\sum_{k=0}^{\infty} \frac{(2 k) ! \Gamma\left(-\frac{1}{2}-y-k\right) \cos [\pi(y+k+1)]}{4^{k} k !}=-\frac{5}{4} \Gamma\left(-\frac{5}{6}\right) \\
\Rightarrow \frac{1}{\pi}=-\frac{4 \Gamma\left(\frac{1}{3}\right)}{5 \Gamma\left(\frac{4}{3}\right) \Gamma\left(\frac{5}{6}\right) \Gamma\left(-\frac{5}{6}\right)} .
\end{gathered}
$$

Let $x=\frac{1}{5}$ and $y=\frac{1}{3}$ in Corollary 2, then

$$
\frac{1}{\pi}=\frac{1}{\cos \left(\frac{8 \pi}{15}\right) \Gamma\left(-\frac{1}{30}\right) \Gamma\left(\frac{31}{30}\right)}=\frac{1}{\left[\frac{1}{8}(1+\sqrt{5})-\frac{1}{4} \sqrt{\frac{3}{2}(5-\sqrt{5})}\right] \Gamma\left(-\frac{1}{30}\right) \Gamma\left(\frac{31}{30}\right)}
$$


and

$$
\frac{1}{\pi}=\frac{-1}{\cos \left(\frac{\pi}{3}\right) \Gamma\left(-\frac{5}{6}\right) \Gamma\left(\frac{11}{6}\right)}=\frac{-2}{\Gamma\left(-\frac{5}{6}\right) \Gamma\left(\frac{11}{6}\right)}
$$

Let $x=\frac{1}{4}$ and $y=\frac{1}{3}$ in Corollary 2, then

$$
\frac{1}{\pi}=\frac{1}{\cos \left(\frac{7 \pi}{12}\right) \Gamma\left(-\frac{1}{12}\right) \Gamma\left(\frac{13}{12}\right)}=\frac{1}{\frac{1-\sqrt{3}}{2 \sqrt{2}} \Gamma\left(-\frac{1}{12}\right) \Gamma\left(\frac{13}{12}\right)}=\frac{2 \sqrt{2}}{(1-\sqrt{3}) \Gamma\left(-\frac{1}{12}\right) \Gamma\left(\frac{13}{12}\right)} .
$$

Corollary 4. For $\mathfrak{R}(x)>0$ and $\mathfrak{R}(y)>0$, then

$$
\frac{\sqrt{\pi} \Gamma(x) \Gamma(y)}{2^{2 x+2 y-1} \Gamma(x+y) \Gamma\left(y+\frac{1}{2}\right)}=\sum_{k=0}^{\infty} \frac{(2 k) ! \Gamma(x+k) \Gamma(x+y+k)}{k !^{2} \Gamma[2(x+y+k)]} .
$$

and

$$
\frac{\sqrt{\pi} \Gamma(y)}{2^{2 y+1} \Gamma(y+1) \Gamma\left(y+\frac{1}{2}\right)}=\sum_{k=0}^{\infty} \frac{(2 k) ! \Gamma(y+k+1)}{k ! \Gamma[2(y+k+1)]}
$$

Proof. In [2, page 887], I have

$$
\Gamma(2 x)=\frac{2^{2 x-1}}{\sqrt{\pi}} \Gamma(x) \Gamma\left(x+\frac{1}{2}\right) \Rightarrow \frac{1}{\Gamma\left(x+\frac{1}{2}\right)}=\frac{2^{2 x-1}}{\sqrt{\pi}} \frac{\Gamma(x)}{\Gamma(2 x)} .
$$

I substitute (9) into Theorem 1 and Corollary 1, this completes the proof. $\square$

Corollary 6. I have

$$
\sqrt{\pi}=2 \Gamma\left(\frac{3}{2}\right)
$$

and

$$
\Gamma\left(\frac{1}{4}\right)=4 \Gamma\left(\frac{5}{4}\right)
$$

Proof. Let $y=\frac{1}{2}$ in Corollary 4 , then

$$
\frac{\sqrt{\pi} \Gamma\left(\frac{1}{2}\right)}{4 \Gamma\left(\frac{3}{2}\right) \Gamma(1)}=\sum_{k=0}^{\infty} \frac{(2 k) ! \Gamma\left(\frac{3}{2}+k\right)}{k ! \Gamma(2 k+3)}=\frac{\sqrt{\pi}}{2} \Rightarrow \sqrt{\pi}=2 \Gamma\left(\frac{3}{2}\right) .
$$

Let $y=\frac{1}{4}$ in Corollary 4 , then

$$
\frac{\sqrt{\pi} \Gamma\left(\frac{1}{4}\right)}{2^{\frac{3}{2}} \Gamma\left(\frac{5}{4}\right) \Gamma\left(\frac{3}{4}\right)}=\sum_{k=0}^{\infty} \frac{(2 k) ! \Gamma\left(\frac{5}{4}+k\right)}{k ! \Gamma\left(\frac{5}{2}+2 k\right)}=\frac{\sqrt{2 \pi}}{\Gamma\left(\frac{3}{4}\right)} \Rightarrow \Gamma\left(\frac{1}{4}\right)=4 \Gamma\left(\frac{5}{4}\right) .
$$


Theorem 2. For $\mathfrak{R}(z)>0$, then

$$
\Gamma(z) \sum_{n=1}^{M} \frac{\Gamma(n)}{\Gamma(z+n) \Gamma\left(n+\frac{1}{2}\right)}=\sum_{k=0}^{\infty} \frac{(2 k) ! \Gamma(z+k)}{4^{k} k !^{2}} \sum_{n=1}^{M} \frac{1}{\Gamma\left(z+n+k+\frac{1}{2}\right)}
$$

and

$$
\frac{\sqrt{\pi} \Gamma(z)}{2^{2 z-1}} \cdot \sum_{n=1}^{M} \frac{\Gamma(n)}{2^{2 n} \Gamma(z+n) \Gamma\left(n+\frac{1}{2}\right)}=\sum_{k=0}^{\infty} \frac{(2 k) ! \Gamma(z+k)}{k !^{2}} \sum_{n=1}^{M} \frac{\Gamma(z+n+k)}{\Gamma[2(z+n+k)]} .
$$

Proof. Let $x=z$ and $y=n$ in Theorem 1 and Corollary 4

$$
\Gamma(z) \frac{\Gamma(n)}{\Gamma(z+n) \Gamma\left(n+\frac{1}{2}\right)}=\sum_{k=0}^{\infty} \frac{(2 k) ! \Gamma(z+k)}{4^{k} k !^{2} \Gamma\left(z+n+k+\frac{1}{2}\right)}
$$

and

$$
\frac{\sqrt{\pi} \Gamma(\mathrm{z})}{2^{2 \mathrm{z}-1}} \cdot \frac{\Gamma(\mathrm{n})}{2^{2 \mathrm{n}} \Gamma(\mathrm{z}+\mathrm{n}) \Gamma\left(\mathrm{n}+\frac{1}{2}\right)}=\sum_{\mathrm{k}=0}^{\infty} \frac{(2 \mathrm{k}) ! \Gamma(\mathrm{z}+\mathrm{k}) \Gamma(\mathrm{x}+\mathrm{n}+\mathrm{k})}{\mathrm{k} !^{2} \Gamma[2(\mathrm{z}+\mathrm{n}+\mathrm{k})]} .
$$

I let the summation from 1 at $M$ to $n$ in (10) and (11)

$$
\Gamma(z) \sum_{n=1}^{M} \frac{\Gamma(n)}{\Gamma(z+n) \Gamma\left(n+\frac{1}{2}\right)}=\sum_{k=0}^{\infty} \frac{(2 k) ! \Gamma(z+k)}{4^{k} k !^{2}} \sum_{n=1}^{M} \frac{1}{\Gamma\left(z+n+k+\frac{1}{2}\right)}
$$

and

$$
\frac{\sqrt{\pi} \Gamma(z)}{2^{2 z-1}} \cdot \sum_{n=1}^{M} \frac{\Gamma(n)}{2^{2 n} \Gamma(z+n) \Gamma\left(n+\frac{1}{2}\right)}=\sum_{k=0}^{\infty} \frac{(2 k) ! \Gamma(z+k)}{k !^{2}} \sum_{n=1}^{M} \frac{\Gamma(z+n+k)}{\Gamma[2(z+n+k)]} .
$$

\section{REFERENCES}

[1] Guedes, Edigles, On the Complete Elliptic Integrals and Babylonian Identity I: The 1/ $\pi$ formulaes Involving Gamma functions and Summations, 2013.

[2] Gradshteyn, I. S. and Ryzhik, I. M., Table of Integrals, Series and Products, Academic Press, 2000. 